Article

\title{
Modification of Polyhedral Oligomeric Silsesquioxanes (POSS) Molecules by Ruthenium Catalyzed Cross Metathesis
}

\author{
Justyna Czaban-Jóźwiak *,+ (D) , Łukasz Woźniak, Artur Ulikowski, Katarzyna Kwiecińska ${ }^{\mathbb{D}}$, \\ Adam A. Rajkiewicz ${ }^{(D)}$ and Karol Grela * \\ Institute of Organic Chemistry, Polish Academy of Sciences, Kasprzaka 44/52, PO Box 58, \\ 01-224 Warsaw, Poland; lukasz.wozniak5@gmail.com (Ł.W.); artur.ulikowski@gmail.com (A.U.); \\ kwiecinskaxyz@gmail.com (K.K.); a.rajkiewicz@cent.uw.edu.pl (A.A.R.) \\ * Correspondence: justyna.czabanjozwiak@kaust.edu.sa (J.C.-J.); karol.grela@gmail.com (K.G.); \\ Tel.: +48-22-632-66-81 (K.G.) \\ + Current address: Functional Material Design, Discovery and Development Research Group (FMD3), \\ Advanced Membranes and Porous Materials Center (AMPMC), Division of Physical Sciences and \\ Engineering (PSE), King Abdullah University of Science and Technology (KAUST), Thuwal 23955-6900, \\ Kingdom of Saudi Arabia
}

Received: 17 June 2018; Accepted: 11 July 2018; Published: 14 July 2018

check for updates

\begin{abstract}
The scope of ruthenium (Ru)-catalyzed cross metathesis (CM) of allyl-decorated polyhedral oligomeric silsesquioxanes (POSS) was explored. A variety of different commercial and non-commercial ruthenium complexes were tested to determine that the nitro-activated $\mathrm{Ru}$ catalyst is optimal for this transformation. The reported transformation was used to prepare selected hybrid steroid-POSS compounds.
\end{abstract}

Keywords: cross metathesis; cross metathesis (CM); steroid; POSS; polyhedral oligomeric silsesquioxanes; ruthenium complexes

\section{Introduction}

Polyhedral oligomeric silsesquioxanes (POSS) are characterized by their remarkable cubic structure, which has received attention from scientists of different disciplines [1-3]. POSS can be used as precursors and components in a variety of hybrid materials for biomedical applications, such as biomedical devices, tissue engineering scaffolds, drug delivery systems, dental applications, and biological sensors [4-10]. The POSS molecule (Figure 1) consists of a cubic silica-oxygen core that is surrounded by eight tunable groups, one in each corner of the cube. These groups can be identical or one of them can be different, allowing for further site selective modification. The chain groups $(R, X)$ can be substituted with a potentially unlimited number of organofunctional groups, such as alkyls, olefins, alcohols, esters, anhydrides, amines, imides, epoxides, thiols, sulfonates, silanols, and many others [11].

Olefin cross metathesis (CM) is one of the most powerful methods for preparing variously substituted alkenes [12-15]. CM is widely used as a key step in the synthesis of many chemicals from simple oils [16,17] to complex structures that exhibit biological activity [18-20].

$\mathrm{CM}$ is catalyzed by ruthenium (Ru) or molybdenum (Mo) complexes; the most common catalysts are ruthenium complexes such as Grubbs (Gr), Hoveyda-Grubbs (Hov) and Indenylidene (Ind) (Figure 2) [21]. 


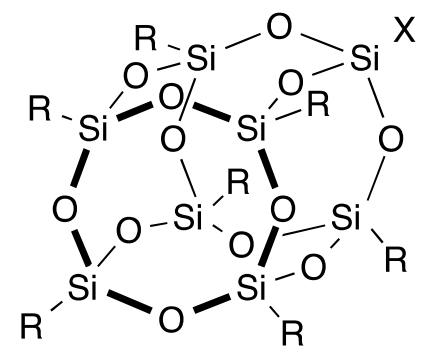

Figure 1. The general features of polyhedral oligomeric silsesquioxanes (POSS).

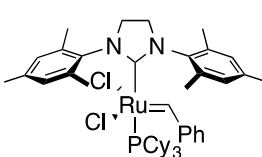

Gr-II

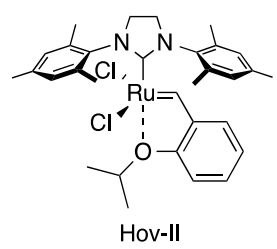

Hov-I

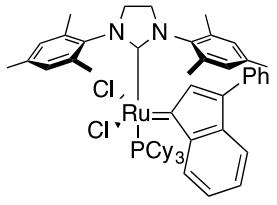

Ind-II

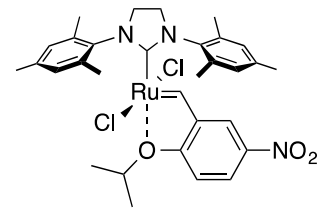

Nitro

Figure 2. Commonly used types of ruthenium catalysts for olefin metathesis.

The first cross metathesis reaction of octavinyl POSS was performed by the Feher group in 1997 employing Schrock and Grubbs catalysts [22]. Schrock catalysts were substantially more active compared to Grubbs. The authors showed that metathesis offers an important new route in the functionalization of POSS derivatives. Even though Schrock catalysts provided better results, the authors emphasized that ruthenium catalysts are the future of POSS CM. Marciniec et al. reported the first efficient CM of vinyl-POSS in the presence of a ruthenium catalyst [23-25]. POSS CM was also applied for the synthesis of POSS-core dendrimers [26,27]. Notably, this methodology required extremely high catalyst loading ( $45 \mathrm{~mol} \%$ ) of first generation Grubbs catalyst (Gr-I) $[26,27]$.

The CM reaction of POSS derivatives might be considered the most direct method for obtaining new POSS biohybrids, which is being intensively explored in nanomedicine [4]. Herein, we report the first examples of allyl-POSS CM reaction. To achieve this transformation, a variety of commercially available ruthenium complexes, as well as a few obtained in our laboratories, were screened in two model CM reactions of allyl-POSS derivative (1), using easily reacting cis-1,4-Diacetoxy-2-butene (3) and the more challenging, electron-deficient, tert-butyl acrylate (2) as CM partners. After selecting the most efficient catalysts the utility of this method was demonstrated by the synthesis of some selected POSS-steroid conjugates.

\section{Results and Discussion}

The first step in the present study was to choose an appropriate catalyst and conditions. For this approach, we selected tert-butyl acrylate (2) and (Z)-diacetoxy-but-2-en (3) as the model CM partners (Table 1). The CM reactions were performed at room temperature in dichloromethane (DCM). Notably, the catalyst loading in these preliminary experiments was set at $0.5 \mathrm{~mol} \%$, which is substantially lower than in the previous reports on CM of POSS molecules of $45 \mathrm{~mol} \%$.

Almost all tested complexes (Figures 2 and 3) presented some activity toward POSS CM. First generation catalysts [Ru]-1 and [Ru]-2 (Table 1, Entry 1 and 2, respectively) showed the lowest activity among the catalysts studied here. In the Indenylidene family, complexes Ind-2 (Table 1, Entry 10) and [Ru]-8 (Table 1, Entry 12) did not catalyse the process, whereas [Ru]-7 (Table 1, Entry 11) exhibited medium activity. However, those complexes perform better at elevated temperatures [28]. Complexes with SIPr N-Heterocyclic Carbene (NHC) ligands [Ru]-4 (Table 1, Entry 5), [Ru]-5 (Table 1, Entry 8), [Ru]-13 (Table 1, Entry 22), and Ru-[14] (Table 1, Entry 23) displayed higher catalytic activity toward the CM reaction in comparison to the parent catalysts [20,29]. Complexes bearing so-called 
"Turbo" unsaturated NHC ligand [Ru]-3 (Table 1, Entry 4) and [Ru]-6 (Table 1, Entry 9) according the literature supposed to behave similarly to SIPr-modified complexes [30]. Interestingly, in this case, we observed lower catalytic activity. A modification of the benzylidene ligand, as in the case of Nitro (Table 1, Entry 13), [Ru]-13 (Table 1, Entry 21), [Ru]-14 (Table 1, Entry 23), and [Ru]-15 [31] (Table 1, Entry 25), did not improve catalytic activity compared to parent Hov-II (Table 1, Entry 10). Similarly, the additional chelation present in the case of complexes [Ru]-9 (Table 1, Entry 15), [Ru]-10 [32,33] (Table 1, Entry 17), and [Ru]-11 (Table 1, Entry 19) did not significantly change the reaction outcome.

Table 1. Cross metathesis of allyl-polyhedral oligomeric silsesquioxanes (POSS) derivative with model partners.
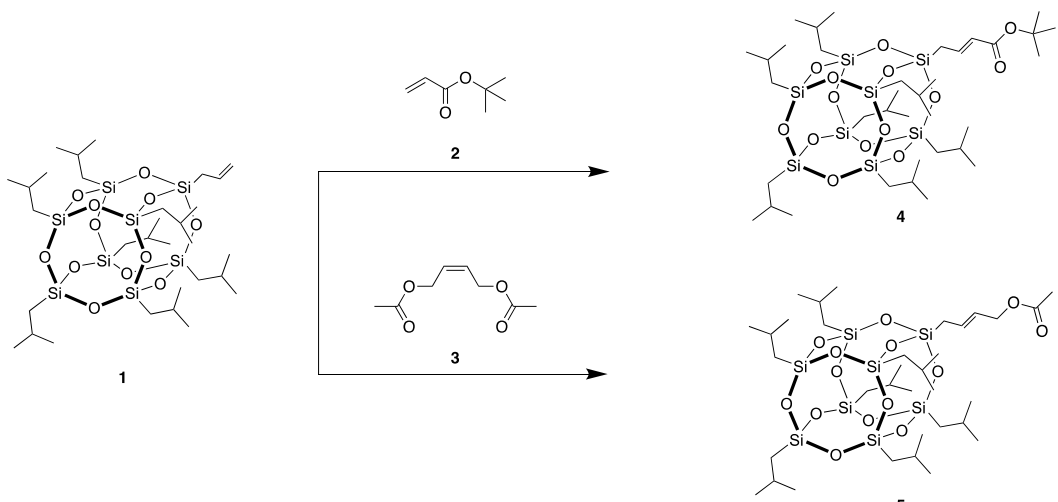

\begin{tabular}{|c|c|c|c|c|}
\hline Entry & Catalyst & Time (h) & Yield of $4(\%)$ & Yield of $5(\%)$ \\
\hline 1 & {$[\mathrm{Ru}]-1$} & 5 & 6 & 33 \\
\hline 2 & {$[\mathrm{Ru}]-2$} & 5 & 5 & 40 \\
\hline 3 & Gr-II & 5 & 71 & 80 \\
\hline 4 & {$[R u]-3$} & 5 & 28 & 31 \\
\hline 5 & {$[\mathrm{Ru}]-4$} & 5 & 79 & 83 \\
\hline 6 & Hov-II & 5 & 99 & 98 \\
\hline 7 & Hov-II & 2 & 80 & 73 \\
\hline 8 & {$[\mathrm{Ru}]-5$} & 5 & 99 & 69 \\
\hline 9 & {$[\mathrm{Ru}]-6$} & 5 & 40 & 57 \\
\hline 10 & Ind-II & 5 & 0 & 0 \\
\hline 11 & {$[\mathrm{Ru}]-7$} & 5 & 50 & 52 \\
\hline 12 & {$[\mathrm{Ru}]-8$} & 5 & 0 & 0 \\
\hline 13 & Nitro & 24 & 90 & 64 \\
\hline 14 & Nitro & 5 & 87 & 76 \\
\hline 15 & {$[\mathrm{Ru}]-9$} & 5 & 94 & 89 \\
\hline 16 & {$[\mathrm{Ru}]-9$} & 2 & 81 & 69 \\
\hline 17 & {$[\mathrm{Ru}]-10$} & 5 & 99 & 66 \\
\hline 18 & {$[\mathrm{Ru}]-10$} & 2 & 72 & 26 \\
\hline 19 & {$[\mathrm{Ru}]-11$} & 5 & 56 & 77 \\
\hline 20 & {$[\mathrm{Ru}]-12$} & 5 & 91 & 82 \\
\hline 21 & [Ru]-12 & 2 & 93 & 90 \\
\hline 22 & {$[\mathrm{Ru}]-13$} & 5 & 91 & 82 \\
\hline 23 & {$[\mathrm{Ru}]-14$} & 5 & 91 & 95 \\
\hline 24 & [Ru]-14 & 2 & 87 & 80 \\
\hline 25 & {$[\mathrm{Ru}]-15$} & 5 & 85 & 83 \\
\hline 26 & {$[\mathrm{Ru}]-16$} & 5 & 81 & 86 \\
\hline 27 & {$[\mathrm{Ru}]-17$} & 5 & 96 & 82 \\
\hline 28 & {$[\mathrm{Ru}]-18$} & 5 & 90 & 82 \\
\hline
\end{tabular}

Reaction conditions: 1 (0.5 mmol), $2(1 \mathrm{mmol})$, or $3(1.5 \mathrm{mmol})$, [Ru $0.5 \mathrm{~mol} \%$, dichloromethane (DCM) $(4 \mathrm{~mL})$, room temperature $(\mathrm{RT})$. 


$$
\begin{gathered}
\mathrm{Cl}_{1} \mathrm{PCy}_{3} \\
\mathrm{Ru} \\
\mathrm{Cl}= \\
\mathrm{PCy} \mathrm{Ph}_{3} \\
{[\mathrm{Ru}]-1}
\end{gathered}
$$

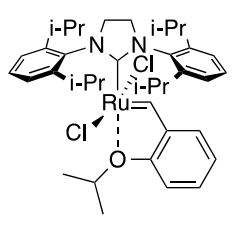

$[\mathrm{Ru}]-5$

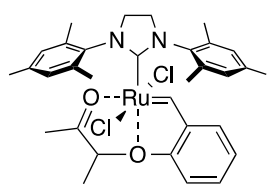

$[\mathrm{Ru}]-9$

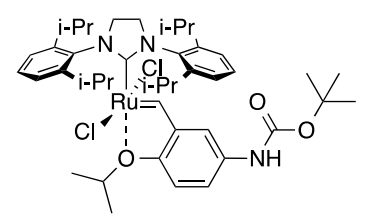

$[\mathrm{Ru}]-13$

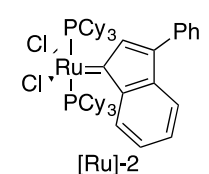

$[\mathrm{Ru}]-2$

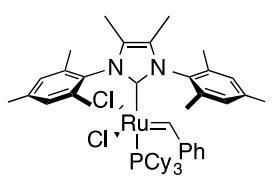

$[\mathrm{Ru}]-3$

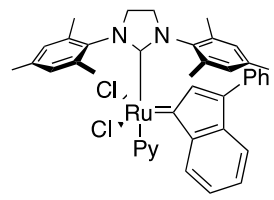

[Ru]-7

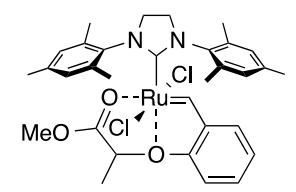

$[\mathrm{Ru}]-10$

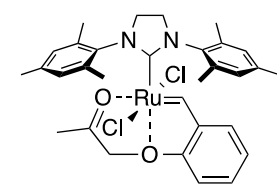

$[\mathrm{Ru}]-11$

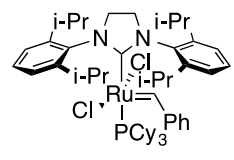

[Ru]-4

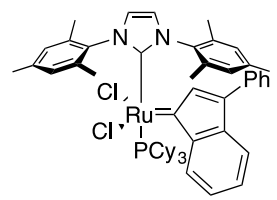

[Ru]-8

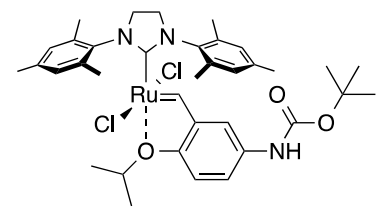

[Ru]-12

Figure 3. Additional ruthenium complexes used in this study.

Next, we investigated the catalytic activity of Ru complexes bearing the NHC ligand with a more bulky naphthyl side chains (Dorta's NHC ligands, Figure 4) [34]. This type of complexes possesses higher activity in some olefin metathesis transformations [35-37]. However, in the case of POSS CM, catalysts [Ru]-16 (Table 1, Entry 26), [Ru]-17 (Table 1, Entry 27), and [Ru]-18 (Table 1, Entry 28) generally displayed similar activity as the other "decorated" Hoveyda-type complexes.

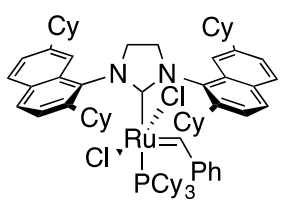

[Ru]-16

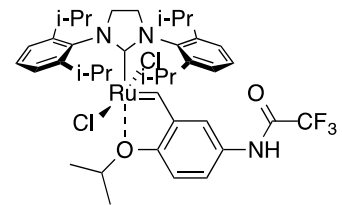

$[\mathrm{Ru}]-14$

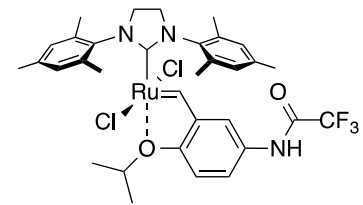

$[\mathrm{Ru}]-15$ 
As these steroidal CM partners can be more challenging in terms of reactivity than a simple diacetate 3 or even acrylate 2 , we expected that an increase in the reaction temperature and catalyst loading might be necessary to obtain practically useful product yields.

Reacting androsterone derivative 6 with allyl-POSS (1) (Table 2) in the previously determined optimal conditions yielded only traces $(3 \%)$ of the desired product. Increasing the temperature to $45^{\circ} \mathrm{C}$ and the loading of Hov-II to $2 \mathrm{~mol} \%$ increased the yield of 7 to $36 \%$. Further improvement was achieved by performing the reaction $100{ }^{\circ} \mathrm{C}$ in toluene to access the desired product 7 in $69 \%$ yield. Finally, when we performed the reaction in the presence of the Nitro catalyst, product 7 was obtained with $72 \%$ of yield as a mixture of $\mathrm{Z} / \mathrm{E}$ isomers $20: 80$.

Table 2. Cross metathesis of allyl-POSS 1 with derivative of androsterone 6 .

\begin{tabular}{ccccc} 
Catalyst (mol\%) & Solvent & Temperature $\left({ }^{\circ} \mathrm{C}\right)$ & Time (h) & Yield of 7 (\%) \\
\hline Hov-II (0.5) & DCM & RT & 5 & 36 \\
Hov-II (2) & DCM & 45 & 5 & 69 \\
Nov-II (2) & Toluene & 100 & 5 & 72 \\
\hline Nitro (2) & Toluene & 100 & 5 & 36 \\
\hline
\end{tabular}

Pregnenolone derivative 8 (Table 3) react with allyl-POSS to give the functionalized POSS 9 in $29 \%$ yield using $0.5 \mathrm{~mol} \%$ of Hov-II at room temperature. Increasing the temperature to $100{ }^{\circ} \mathrm{C}$ did not significantly improve the yield (36\%), as well as applying Nitro as a catalyst (46\%). However, the best result was obtained refluxing the reaction mixture in DCM for $24 \mathrm{~h}$ using $2 \mathrm{~mol} \%$ of Nitro catalyst. In these conditions, the desired product 9 was obtained in 69\% yield as a mixture of $\mathrm{Z} / \mathrm{E}$ isomers 20:80

Table 3. Cross metathesis of allyl-POSS 1 with derivative of pregnenolone 8.

\begin{tabular}{ccccc} 
& & & \\
\hline Catalyst (mol\%) & Solvent & Temperature $\left({ }^{\circ} \mathrm{C}\right)$ & Time (h) & Yield of 9 (\%) \\
\hline Hov-II (0.5) & DCM & RT & 5 & 29 \\
Hov-II (2) & Toluene & 100 & 5 & 46 \\
Nitro (2) & Toluene & 100 & 10 & 69 \\
Nitro (2) & DCM & 45 & 24 & 9 \\
\hline
\end{tabular}

We were intrigued by very similar results obtained at room temperature and $100{ }^{\circ} \mathrm{C}$ using substrate 8. We hypothesized that the higher reaction temperature might facilitate both reaction between and 1 and 8 but also self CM of $\mathbf{1}$. We performed control experiments by mixing $\mathbf{1}$ with $0.5 \mathrm{~mol} \%$ of Hov-II in toluene at $100{ }^{\circ} \mathrm{C}$ and in DCM at room temperature. Indeed, the experiment at elevated temperature resulted in $90 \%$ of the self cross metathesis product and no conversion was observed at room temperature.

Next, we employed the optimal conditions for substrate 8 to prepare estrone POSS derivative 11 (Scheme 1) that was obtained as 20:80 mixture of $\mathrm{Z} / \mathrm{E}$ isomers in 62\% yield. 


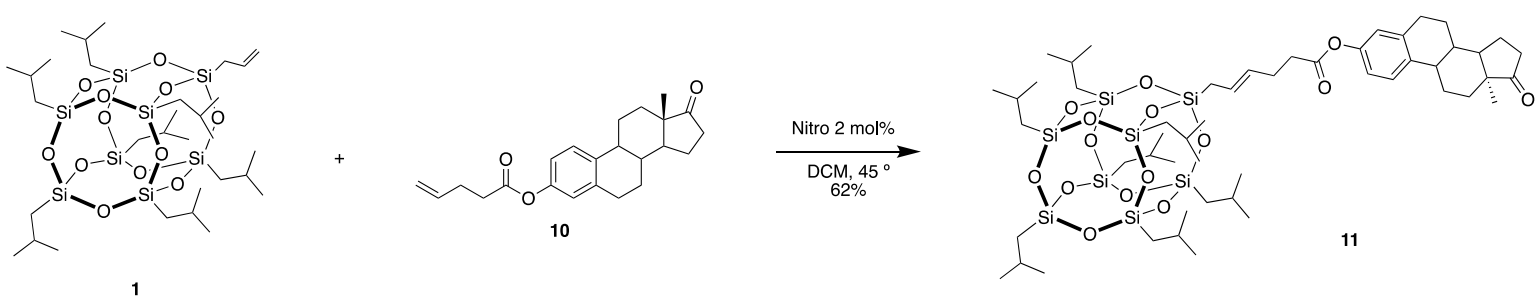

Scheme 1. Cross metathesis of allyl-POSS 1 with the derivative of estrone $\mathbf{1 0 .}$

\section{Materials and Methods}

\subsection{General}

Nuclear magnetic resonance (NMR) spectra were recorded in $\mathrm{CDCl}_{3}$ (chloroform- $\mathrm{d}$ ) or benzene- $\mathrm{d}_{6}$ solutions (unless indicated otherwise); chemical shifts are reported in the $\delta$ scale in ppm, with the solvent signal as the internal standard $\left(\mathrm{CDCl}_{3},{ }^{1} \mathrm{H}\right.$ NMR $7.26 \mathrm{ppm} ;{ }^{13} \mathrm{C}$ NMR 77.00 ppm, benzene- $\mathrm{d}_{6}$, ${ }^{1} \mathrm{H}$ NMR $7.16 \mathrm{ppm},{ }^{13} \mathrm{C}$ NMR $\left.128.06 \mathrm{ppm}\right)$. Column chromatography was performed on Merck silica gel 60, 230-400 mesh (Darmstadt, Germany). Thin layer chromatography (TLC) was performed on aluminum sheets, Merck $60 \mathrm{~F}_{254}$ (Darmstadt, Germany). Anhydrous solvents were obtained by distillation over calcium chloride $\left(\mathrm{CaCl}_{2}\right)(\mathrm{DCM}$, Toluene). All reactions were performed under argon (Ar) in pre-dried glassware using Schlenk techniques.

\subsection{General Procedure for the Cross Metathesis of $\mathbf{1}$ with Different Partners}

The catalyst was added in one portion to a solution of propyl-POSS and appropriate partner in DCM/Toluene $(4 \mathrm{~mL})$. The resulting mixture was stirred in an appropriate temperature under an argon atmosphere. The solvent was removed under reduced pressure. The crude product was purified by flash chromatography (c-hexane/EtOAc) to obtain pure product.

\subsection{4-(POSS)but-2-en-1-yl acetate (4)}

${ }^{1} \mathrm{H}$ NMR (500 MHz, $\left.\mathrm{CDCl}_{3}\right): \delta, 5.79-5.70(\mathrm{~m}, 1 \mathrm{H}), 5.66(\mathrm{dtt}, J=11.4,8.6,1.4 \mathrm{~Hz}, 1 \mathrm{H}), 5.55-5.45$ $(\mathrm{m}, 1 \mathrm{H}), 4.61(\mathrm{dd}, J=6.8,1.4 \mathrm{~Hz}, 1 \mathrm{H}), 4.48(\mathrm{dd}, J=6.7,1.1 \mathrm{~Hz}, 2 \mathrm{H}), 2.06(\mathrm{~s}, 1 \mathrm{H}), 2.04(\mathrm{~s}, 2 \mathrm{H}), 1.85(\mathrm{dpd}$, $J=13.4,6.7,2.3 \mathrm{~Hz}, 7 \mathrm{H}), 1.66(\mathrm{dd}, J=8.6,1.4 \mathrm{~Hz}, 1 \mathrm{H}), 1.61(\mathrm{dd}, J=7.9,1.3 \mathrm{~Hz}, 2 \mathrm{H}), 0.96(\mathrm{dd}, J=6.6$, $1.0 \mathrm{~Hz}, 42 \mathrm{H}), 0.63-0.58(\mathrm{~m}, 14 \mathrm{H}) \mathrm{ppm} .{ }^{13} \mathrm{C} \mathrm{NMR}\left(126 \mathrm{MHz}, \mathrm{CDCl}_{3}\right): \delta, 171.1,171.0,130.7,128.6,124.2$, 122.9, 77.4, 77.3, 77.1, 76.9, 65.6, 60.6, 25.9, 25.8, 25.8, 24.0, 22.9, 22.6, 22.5, 21.2, 18.4, 14.6, 1.2, 0.1 ppm (Supplementary Materials). IR (film, DCM) v 2954, 2927, 2907, 2870, 1745, 1465, 1401, 1383, 1366, 1332, 1230, 1169, 1107, 1038, 964, 838. EA Anal. Calcd. For $\mathrm{C}_{34} \mathrm{H}_{72} \mathrm{O}_{14} \mathrm{Si}_{8}$ : C, 43.93; H, 7.81; found C, 43.77; $\mathrm{H}$, 7.98. MS (ESI): $m / z[\mathrm{M}+\mathrm{Na}]^{+}: 951$.

\section{4. tert-butyl 4-(POSS)but-2-enoate (5)}

${ }^{1} \mathrm{H}$ NMR (500 MHz, $\left.\mathrm{CDCl}_{3}\right): \delta, 6.86(\mathrm{dt}, J=15.4,8.5 \mathrm{~Hz}, 1 \mathrm{H}), 5.67(\mathrm{dt}, J=15.4,1.4 \mathrm{~Hz}, 1 \mathrm{H}), 1.85$ $(\mathrm{dpd}, J=13.5,6.8,1.5 \mathrm{~Hz}, 7 \mathrm{H}), 1.75(\mathrm{dd}, J=8.5,1.4 \mathrm{~Hz}, 2 \mathrm{H}), 1.47(\mathrm{~s}, 9 \mathrm{H}), 0.95(\mathrm{dd}, J=6.6,1.0 \mathrm{~Hz}, 43 \mathrm{H})$, 0.63-0.58 (m, 14H) ppm. ${ }^{13} \mathrm{C}$ NMR (126 MHz, $\left.\mathrm{CDCl}_{3}\right): \delta, 166.1,143.0,123.3,79.8,79.7,28.5,28.4,25.9$, 25.8, 24.1, 24.0, 24.0, 22.7, 22.6, 22.5, 22.2, 22.1, 19.4, 1.2, 0.2 ppm (Supplementary Materials). IR (film, DCM) v 2955, 2932, 2907, 2870, 1714, 1646, 1465, 1401, 1383, 1367, 1326, 1296, 1230, 1213, 1168, 1106, 1040, 982, 839. EA Anal. Calcd. For $\mathrm{C}_{36} \mathrm{H}_{76} \mathrm{O}_{14} \mathrm{Si}_{8}$ : C, 45.15; H, 8.00; found C, 45.03; H, 7.93. MS (ESI): $m / z[\mathrm{M}+\mathrm{Na}]^{+}: 979$.

\section{5. (10R,13S)-10,13-dimethyl-1,2,3,4,7,8,9,10,11,12,13,14,15,16-tetradecahydrospiro[cyclopenta[a]} phenanthrene-17,2'-[1,3]dioxolan]-3-yl 6-(POSS)hex-4-enoate (7)

${ }^{1} \mathrm{H}$ NMR $\left(400 \mathrm{MHz}, \mathrm{C}_{6} \mathrm{D}_{6}\right): \delta, 5.76-5.62(\mathrm{~m}, 1 \mathrm{H}), 5.59-5.44(\mathrm{~m}, 1 \mathrm{H}), 5.35-5.26(\mathrm{~m}, 1 \mathrm{H}), 4.87$ (ddt, $J=11.1,8.4,5.5 \mathrm{~Hz}, 1 \mathrm{H}), 3.61-3.46(\mathrm{~m}, 4 \mathrm{H}), 2.58-2.46(\mathrm{~m}, 1 \mathrm{H}), 2.41(\mathrm{t}, J=6.9 \mathrm{~Hz}, 2 \mathrm{H}), 2.36-2.30(\mathrm{~m}, 1 \mathrm{H})$, 
2.18-1.98 (m, 9H), $1.88(\mathrm{dddd}, J=17.2,14.9,7.3,4.0 \mathrm{~Hz}, 4 \mathrm{H}), 1.80-1.69(\mathrm{~m}, 3 \mathrm{H}), 1.67-1.53(\mathrm{~m}, 5 \mathrm{H}), 1.46$ $(\mathrm{dd}, J=11.4,3.2 \mathrm{~Hz}, 3 \mathrm{H}), 1.36(\mathrm{td}, J=13.8,13.0,5.0 \mathrm{~Hz}, 3 \mathrm{H}), 1.16-1.04(\mathrm{~m}, 47 \mathrm{H}), 0.96(\mathrm{~d}, J=1.6 \mathrm{~Hz}, 3 \mathrm{H})$, $0.91(\mathrm{t}, J=2.3 \mathrm{~Hz}, 3 \mathrm{H}), 0.88-0.80(\mathrm{~m}, 14 \mathrm{H}) \mathrm{ppm} .{ }^{13} \mathrm{C} \mathrm{NMR}\left(101 \mathrm{MHz}, \mathrm{CDCl}_{3}\right): \delta, 171.9,139.8,129.8$, 124.6, 122.7, 119.6, 73.8, 65.2, 64.6, 50.9, 50.3, 46.1, 38.8, 37.2, 36.9, 34.9, 34.7, 32.4, 31.7, 30.9, 28.8, 28.3, 25.9, 24.5, 23.2, 23.1, 22.9, 20.9, 19.4, 18.5, 14.6 ppm (Supplementary Materials). IR (film, DCM) v 3444, 2925, 2856, 1733, 1671, 1462, 1378, 1307, 1255, 1228, 1170, 1109, 1040, 959, 903, 881.

3.6. (13S)-13-methyl-17-oxo-7,8,9,11,12,13,14,15,16,17-decahydro-6H-cyclopenta[a] phenanthren-3-yl-6-(POSS)hex-4-enoate (9)

${ }^{1} \mathrm{H}$ NMR $\left(400 \mathrm{MHz}, \mathrm{C}_{6} \mathrm{D}_{6}\right):$, 5.94-5.82 (m, $\left.0.2 \times 1 \mathrm{H}\right), 5.76-5.65(\mathrm{~m}, 1 \mathrm{H}), 5.62-5.52(\mathrm{~m}, 0.8 \times 1 \mathrm{H})$, $5.35(\mathrm{dt}, J=5.6,1.8 \mathrm{~Hz}, 1 \mathrm{H}), 3.45-3.34(\mathrm{~m}, 1 \mathrm{H}), 2.30-2.21(\mathrm{~m}, 2 \mathrm{H}), 2.20-2.03(\mathrm{~m}, 9 \mathrm{H}), 1.99-1.87(\mathrm{~m}, 2 \mathrm{H})$, $1.86-1.77(\mathrm{~m}, 2 \mathrm{H}), 1.75-1.65(\mathrm{~m}, 3 \mathrm{H}), 1.63-1.53(\mathrm{~m}, 2 \mathrm{H}), 1.50-1.36(\mathrm{~m}, 5 \mathrm{H}), 1.31(\mathrm{~s}, 3 \mathrm{H}), 1.14-1.06$ $(\mathrm{m}, 44 \mathrm{H}), 0.96(\mathrm{~s}, 3 \mathrm{H}), 0.93(\mathrm{~s}, 3 \mathrm{H}), 0.89-0.83(\mathrm{~m}, 14 \mathrm{H}) \mathrm{ppm} .{ }^{13} \mathrm{C}$ NMR $\left(101 \mathrm{MHz}, \mathrm{C}_{6} \mathrm{D}_{6}\right): \delta, 141.3,126.8$, 125.6, 121.6, 74.9, 74.8, 74.3, 71.7, 59.2, 58.9, 58.8, 57.3, 50.6, 50.6, 47.6, 43.3, 43.1, 43.1, 42.9, 41.4, 40.7, $40.6,37.7,36.8,32.3,32.2,31.7,27.8,27.2,27.0,26.0,26.0,25.9,24.5,24.4,24.3,24.2,23.1,23.0,22.8,22.5$, 21.4, 21.4, 19.5, 18.6, 14.5, 14.0, 13.9, 13.8 ppm (Supplementary Materials). IR (film, DCM) v 3387, 2954, $2871,1708,1465,1401,1382,1366,1333,1229,1110,954,837$. MS (TOF ES): $m / z[\mathrm{M}+\mathrm{Na}]^{+}: 1210.53$

3.7. ((13S,17R)-13-methyl-17-(4-(POSS)but-2-en-1-yl)-7,8,9,11,12,13,14,15,16,17-decahydro-6Hcyclopenta[a]phenanthrene-3,17-diol (11)

${ }^{1} \mathrm{H}$ NMR $\left(400 \mathrm{MHz}, \mathrm{CDCl}_{3}\right): \delta, 7.30-7.26(\mathrm{~m}, 1 \mathrm{H}), 6.88-6.77(\mathrm{~m}, 2 \mathrm{H}), 5.69-5.56(\mathrm{~m}, 0.2 \times 1 \mathrm{H})$, 5.63-5.55 (m, $0.2 \times 1 \mathrm{H}), 5.54-5.45(\mathrm{~m}, 0.8 \times 1 \mathrm{H}), 5.44-5.35(\mathrm{~m}, 0.8 \times 1 \mathrm{H}), 2.96-2.84(\mathrm{~m}, 2 \mathrm{H}), 2.62-2.54$ $(\mathrm{m}, 2 \mathrm{H}), 2.53-2.46(\mathrm{~m}, 1 \mathrm{H}), 2.46-2.36(\mathrm{~m}, 2 \mathrm{H}), 2.29(\mathrm{t}, J=10.9 \mathrm{~Hz}, 1 \mathrm{H}), 2.21-2.07(\mathrm{~m}, 1 \mathrm{H}), 2.07-1.93$ $(\mathrm{m}, 3 \mathrm{H}), 1.86(\mathrm{dpd}, J=13.4,6.7,2.0 \mathrm{~Hz}, 8 \mathrm{H}), 1.69-1.58(\mathrm{~m}, 3 \mathrm{H}), 1.58-1.38(\mathrm{~m}, 6 \mathrm{H}), 1.05-0.83(\mathrm{~m}, 46 \mathrm{H})$, 0.65-0.56 (m, 14H) ppm. ${ }^{13} \mathrm{C}$ NMR $\left(101 \mathrm{MHz}, \mathrm{CDCl}_{3}\right): \delta, 220.9,172.2,148.7,138.1,137.4,128.2,126.7$, $126.5,125.3,124.8,121.7,121.7,118.9,118.8,50.6,48.1,44.3,38.1,36.0,34.7,34.5,31.7,29.5,28.4,26.5$, 25.9, 25.8, 24.0, 23.9, 23.9, 22.7, 22.6, 22.6, 22.5, 21.7, 18.2, 13.9, 1.2 ppm (Supplementary Materials). IR (film, DCM) v 3455, 3077, 2952, 2928, 2870, 1739, 1641, 1608, 1583, 1494, 1453, 1417, 1369, 1335, 1260, 1226, 1114, 1009, 915. MS (TOF ES): $m / z[\mathrm{M}+\mathrm{Na}]^{+}: 1204.44$

\section{Conclusions}

In summary, we explored the ruthenium-catalyzed cross metathesis reactions between allyl-POSS and five alkenes, including three steroid derivatives. We showed that, even for such challenging partners and relatively mild conditions, such as $0.5-2 \mathrm{~mol} \%$ of catalyst at DCM or toluene at RT to $100{ }^{\circ} \mathrm{C}$, are sufficient to perform an efficient $\mathrm{CM}$ reaction with POSS derivatives. The best catalysts for these transformations were Hov-II and Nitro complexes.

Supplementary Materials: The following are available online. Figure S1: ${ }^{1} \mathrm{H}$ NMR of compound 4; Figure S2: ${ }^{13} \mathrm{C}$ NMR of compound 4; Figure S3: ${ }^{1} \mathrm{H}$ NMR of compound 5; Figure S4: ${ }^{13} \mathrm{C}$ NMR of compound 5; Figure S5: ${ }^{1} \mathrm{H}$ NMR of compound 7; Figure S6: ${ }^{13} \mathrm{C}$ NMR of compound 7; Figure S7: ${ }^{1} \mathrm{H}$ NMR of compound 9; Figure S8: ${ }^{13} \mathrm{C}$ NMR of compound 9; Figure S9: ${ }^{1} \mathrm{H}$ NMR of compound 11; Figure S10: ${ }^{1} \mathrm{H}$ NMR of compound 11.

Author Contributions: Methodology: J.C.J., Ł.W.; Formal Analysis, J.C.J., Ł.W.; Investigation, J.C.J., Ł.W., A.U., K.K., A.A.R.; Writing: original draft preparation, J.C.J.; Writing: review and editing, J.C.J., Ł.W. and K.G.

Funding: This research was funded by The National Science Center, Poland grant number 2011/01/N/ST5/05638.

Acknowledgments: The authors would like to thank Erik Abbenhuis and Gijsbert Gerritsen from Hybrid Catalysis BV for their generous donation of the POSS derivatives used in this studies.

Conflicts of Interest: The authors declare no conflict of interest. 


\section{References}

1. Hartmann-Thompson, C. Applications of polyhedral oligomeric silsesquioxanes; Springer: Dordrecht, The Netherlands, 2011; p. 420.

2. Markovic, E.; Constantopolous, K.; Matisons, J.G. Polyhedral oligomeric silsesquioxanes: From early and strategic development through to materials application. Appl. Polyhedr. Oligomeric Silsesquioxanes 2011, 3, 1-46.

3. Pielichowski, K.; Njuguna, J.; Janowski, B.; Pielichowski, J. Polyhedral oligomeric silsesquioxanes (POSS)-containing nanohybrid polymers. In Supramolecular polymers polymeric betains oligomers; Donnio, B., Guillon, D., Harada, A., Hashidzume, A., Jaeger, W., Janowski, B., Kudaibergenov, S., Laschewsky, A., Njuguna, J., Pielichowski, J., et al., Eds.; Springer: Berlin, Germany, 2006; Volume 201, pp. 225-296.

4. Ghanbari, H.; Cousins, B.G.; Seifalian, A.M. A nanocage for nanomedicine: Polyhedral oligomeric silsesquioxane (POSS). Macromol. Rapid Commun. 2011, 32, 1032-1046. [CrossRef] [PubMed]

5. John, Ł.; Malik, M.; Janeta, M.; Szafert, S. First step towards a model system of the drug delivery network based on amide-POSS nanocarriers. RSC Adv. 2017, 7, 8394-8401. [CrossRef]

6. John, L.; Janeta, M.; Szafert, S. Synthesis of cubic spherosilicates for self-assembled organic-inorganic biohybrids based on functionalized methacrylates. New J. Chem. 2018, 42, 39-47. [CrossRef]

7. Janeta, M.; John, L.; Ejfler, J.; Lis, T.; Szafert, S. Multifunctional imine-POSS as uncommon 3D nanobuilding blocks for supramolecular hybrid materials: Synthesis, structural characterization, and properties. Dalton Trans. 2016, 45, 12312-12321. [CrossRef] [PubMed]

8. Janeta, M.; John, L.; Ejfler, J.; Szafert, S. High-yield synthesis of amido-functionalized polyoctahedral oligomeric silsesquioxanes by using acyl chlorides. Chemistry-a Eur. J. 2014, 20, 15966-15974. [CrossRef] [PubMed]

9. John, Ł. Selected developments and medical applications of organic-inorganic hybrid biomaterials based on functionalized spherosilicates. Mater. Sci. Eng. C 2018, 88, 172-181. [CrossRef] [PubMed]

10. Kannan, R.Y.; Salacinski, H.J.; Butler, P.E.; Seifalian, A.M. Polyhedral oligomeric silsesquioxane nanocomposites: The next generation material for biomedical applications. Account. Chem. Res. 2005, 38, 879-884. [CrossRef] [PubMed]

11. Wu, J.; Mather, P.T. POSS polymers: Physical properties and biomaterials applications. Polym. Rev. 2009, 49, 25-63. [CrossRef]

12. Blackwell, H.E.; O’Leary, D.J.; Chatterjee, A.K.; Washenfelder, R.A.; Bussmann, D.A.; Grubbs, R.H. New Approaches to Olefin Cross-Metathesis. J. Am. Chem. Soc. 2000, 122, 58-71. [CrossRef]

13. Vernall, A.J.; Abell, A.D. Cross metathesis of nitrogen-containing systems. Aldrichimica Acta 2003, 36, 93-105. [CrossRef]

14. Chatterjee, A.K.; Choi, T.-L.; Sanders, D.P.; Grubbs, R.H. A general model for selectivity in olefin cross metathesis. J. Am. Chem. Soc. 2003, 125, 11360-11370. [CrossRef] [PubMed]

15. Zukowska, K.; Grela, K. Cross metathesis. In Olefin Metathesis: Theory and Practice; Grela, K., Ed.; John Wiley \& Sons: Hoboken, NJ, USA, 2014; pp. 39-83, (and citation therein).

16. Czaban, J.; Schertzer, B.M.; Grela, K. Low catalyst loadings in self-metathesis of 1-dodecene. Adv. Synth. Catal. 2013, 355, 1997-2006. [CrossRef]

17. Kajetanowicz, A.; Sytniczuk, A.; Grela, K. Metathesis of renewable raw materials-influence of ligands in the indenylidene type catalysts on self-metathesis of methyl oleate and cross-metathesis of methyl oleate with (Z)-2-butene1,4-diol diacetate. Green Chem. 2014, 16, 1579-1585. [CrossRef]

18. Olszewski, T.K.; Figlus, M.; Bieniek, M. Olefin metathesis: A versatile synthetic tool for use in preparation of active pharmaceutical ingredients. Chimica Oggi-Chemistry Today 2014, 32, 22-29. [CrossRef]

19. Pederson, R.L.; Fellows, I.M.; Ung, T.A.; Ishihara, H.; Hajela, S.P. Applications of olefin cross metathesis to commercial products. Adv. Synth. Catal. 2002, 344, 728-735. [CrossRef]

20. Kirschning, A.; Harmrolfs, K.; Mennecke, K.; Messinger, J.; Schön, U.; Grela, K. Homo- and heterogeneous Ru-based metathesis catalysts in cross-metathesis of 15-allylestrone-Towards 17 $\beta$-hydroxysteroid dehydrogenase type 1 inhibitors. Tetrahedron Lett. 2008, 49, 3019-3022. [CrossRef]

21. Bieniek, M.; Kołoda, D.; Grela, K. A highly selective synthesis of dialkenyl sulfones via cross-metathesis of divinyl sulfone. Org. Lett. 2006, 8, 5689-5692. [CrossRef] [PubMed] 
22. Feher, F.J.; Soulivong, D.; Eklund, A.G.; Wyndham, K.D. Cross-metathesis of alkenes with vinyl-substituted silsesquioxanes and spherosilicates: A new method for synthesizing highly-functionalized Si/O frameworks. Chem. Commun. 1997, 1185-1186. [CrossRef]

23. Itami, Y.; Marciniec, B.; Kubicki, M. Functionalization of octavinylsilsesquioxane by ruthenium-catalyzed silylative coupling versus cross-metathesis. Chemistry - A Eur. J. 2004, 10, 1239-1248. [CrossRef] [PubMed]

24. Rogalski, S.; Pietraszuk, C.; Marciniec, B. Synthesis of siloxy-modified second generation Hoveyda-Grubbs catalysts and their catalytic activity. J. Organomet. Chem. 2009, 694, 3918-3922. [CrossRef]

25. Żak, P.; Pietraszuk, C.; Marciniec, B.; Spólnik, G.; Danikiewicz, W. Efficient functionalisation of cubic monovinylsilsesquioxanes via cross-metathesis and silylative coupling with olefins in the presence of ruthenium complexes. Adv. Synth. Catal. 2009, 351, 2675-2682. [CrossRef]

26. Vautravers, N.R.; Andre, P.; Slawin, A.M.Z.; Cole-Hamilton, D.J. Synthesis and characterization of photoluminescent vinylbiphenyl decorated polyhedral oligomeric silsesquioxanes. Org. Biomol. Chem. 2009, 7, 717-724. [CrossRef] [PubMed]

27. Vautravers, N.R.; Andre, P.; Cole-Hamilton, D.J. Fluorescence activation of a polyhedral oligomeric silsesquioxane in the presence of reducing agents. J. Mater. Chem. 2009, 19, 4545-4550. [CrossRef]

28. Bieniek, M.; Michrowska, A.; Usanov, D.L.; Grela, K. In an attempt to provide a user's guide to the galaxy of benzylidene, alkoxybenzylidene, and indenylidene ruthenium olefin metathesis catalysts. Chem. Eur. J. 2008, 14, 806-818. [CrossRef] [PubMed]

29. Dinger, M.B.; Mol, J.C. High turnover numbers with ruthenium-based metathesis catalysts. Adv. Synth. Catal. 2002, 344, 671-677. [CrossRef]

30. Michael, G.; Soley, K.; Wilson, T.; Christina, O.; Joachim, R. Development of commercially viable thermomorphic catalysts for controlled free radical polymerization. In Catalysis of organic reactions, 1st ed.; Prunier, M.L., Ed.; CRC Press: Boca Raton, FL, USA, 2008; pp. 319-328.

31. Borré, E.; Caijo, F.; Crévisy, C.; Mauduit, M. New library of aminosulfonyl-tagged Hoveyda-Grubbs type complexes: Synthesis, kinetic studies and activity in olefin metathesis transformations. Beilstein J. Org. Chem. 2010, 6, 1159-1166. [CrossRef] [PubMed]

32. Bieniek, M.; Bujok, R.; Cabaj, M.; Lugan, N.; Lavigne, G.; Arlt, D.; Grela, K. Advanced fine-tuning of Grubbs/Hoveyda olefin metathesis catalysts: A further step toward an optimum balance between antinomic properties. J. Am. Chem. Soc. 2006, 128, 13652-13653. [CrossRef] [PubMed]

33. Bieniek, M.; Samojłowicz, C.; Sashuk, V.; Bujok, R.; Śledź, P.; Lugan, N.1.; Lavigne, G.; Arlt, D.; Grela, K. Rational design and evaluation of upgraded Grubbs/Hoveyda olefin metathesis catalysts: Polyfunctional benzylidene ethers on the test bench. Organometallics 2011, 30, 4144-4158. [CrossRef]

34. Luan, X.J.; Mariz, R.; Gatti, M.; Costabile, C.; Poater, A.; Cavallo, L.; Linden, A.; Dorta, R. Identification and characterization of a new family of catalytically highly active imidazolin-2-ylidenes. J. Am. Chem. Soc. 2008, 130, 6848-6858. [CrossRef] [PubMed]

35. Vieille-Petit, L.; Luan, X.; Gatti, M.; Blumentritt, S.; Linden, A.; Clavier, H.; Nolan, S.P.; Dorta, R. Improving grubbs' II type ruthenium catalysts by appropriately modifying the $\mathrm{N}$-heterocyclic carbene ligand. Chem. Commun. 2009, 3783-3785. [CrossRef] [PubMed]

36. Gatti, M.; Vieille-Petit, L.; Luan, X.; Mariz, R.; Drinkel, E.; Linden, A.; Dorta, R. Impact of NHC ligand conformation and solvent concentration on the ruthenium-catalyzed ring-closing metathesis reaction. J. Am. Chem. Soc. 2009, 131, 9498-9499. [CrossRef] [PubMed]

37. Vieille-Petit, L.; Clavier, H.; Linden, A.; Blumentritt, S.; Nolan, S.P.; Dorta, R. Ruthenium olefin metathesis catalysts with N-heterocyclic carbene ligands bearing n-naphthyl side chains. Organometallics 2010, 29, 775-788. [CrossRef]

38. Samojlowicz, C.; Bieniek, M.; Zarecki, A.; Kadyrov, R.; Grela, K. The doping effect of fluorinated aromatic hydrocarbon solvents on the performance of common olefin metathesis catalysts: Application in the preparation of biologically active compounds. Chem. Commun. 2008, 6282-6284. [CrossRef] [PubMed]

Sample Availability: Not available.

(C) 2018 by the authors. Licensee MDPI, Basel, Switzerland. This article is an open access article distributed under the terms and conditions of the Creative Commons Attribution (CC BY) license (http://creativecommons.org/licenses/by/4.0/). 